\title{
Georges Saumon : adieu le physiologiste !
}

\section{In Memoriam: Georges Saumon}

\section{Dreyfuss}

(C) SRLF et Lavoisier SAS 2019

\section{Georges Saumon est mort en mai.}

$\mathrm{Au}$ nom de tous ses élèves et grâce à la suggestion d'Alain Mercat, président de la SRLF, et l'acceptation de Pierre-Emmanuel Charles, rédacteur en chef de Médecine intensive-réanimation, que je souhaite remercier tous deux du fond du cœur, il m'est possible d'écrire quelques lignes à sa mémoire. Certaines des phrases de ce texte avaient été publiées à l'occasion d'un article dans notre revue [1].

Les mots ne peuvent décrire la collaboration extraordinaire dont j'ai eu la chance de bénéficier avec Georges Saumon, médecin, physiologiste, épistémologue, informaticien et bricoleur hors normes. J'ai rencontré Georges pendant mon dernier semestre d'internat que j'effectuais chez Claude Amiel à Bichat. Claude Amiel m'avait encouragé à travailler dans le laboratoire de Guy Basset qui s'intéressait à la mesure de l'eau pulmonaire extravasculaire. Guy Basset avait été très intrigué par les résultats que j'avais obtenus en tentant de reproduire les expériences princeps de Webb et Tierney qui les premiers décrivirent la survenue d'un œdème pulmonaire lors de la ventilation à haute pression et à haut volume [2]. Il m'avait donc suggéré de montrer mes données à Georges en me disant en substance : "il risque d'être un peu critique, voire négatif au début, mais si ça lui plaît il vous aidera beaucoup ». Je n'ai été déçu sur aucun des aspects ! La collaboration avec Georges était un plaisir intellectuel incroyable, et $\mathrm{j}$ 'ai rarement autant ri que lorsque nous faisions les «manips » ensemble, et également lorsque nous écrivions nos papiers en vociférant chaque fois (c'està-dire souvent) que nous n'étions pas d'accord sur une interprétation ou tout simplement sur une phrase, jusqu'à ce que nous tombions d'accord après un vacarme de plusieurs minutes qui indisposait quelque peu les autres collègues de l'unité de recherche...

Nous étions exactement ce que Donald Tierney a écrit de sa collaboration avec Herb Webb, lorsque 30 ans après son

\footnotetext{
D. Dreyfuss $(\bowtie)$

Hôpital Louis-Mourier,

178 , rue des Renouillers,

F-92700 Colombes, France

e-mail : didier.dreyfuss@lmr.aphp.fr
}

article initial il décrivit les conditions de l'élaboration de cette recherche qui allait bouleverser les conceptions sur la ventilation artificielle : «We were an unlikely team » [3].

La photo ci-après (Fig. 1) prise à la grande époque de ma collaboration avec Georges, lors d'un dîner chez moi avec des collègues nord-américains (dont Arthur Slutsky et Alan Morris), explique ce caractère unlikely et témoigne à la fois de cette joie de travailler ensemble et du temps qui passe. Elle évoque ce qui était écrit sur certains cadrans solaires : «Vulnerant omnes, ultima necat». Récemment, ce fut donc la dernière pour Georges.

Sans Georges, aucun des travaux expérimentaux que j'ai eu la chance de pouvoir mener à bien n'aurait vu le jour.

Ses plus proches élèves, Jean-Damien Ricard, Damien Roux, Stéphane Gaudry et Nicolas de Prost, ont partagé cette chance inouïe et lui doivent également beaucoup. Les phrases qui suivent sont les leurs, écrites pour son enterrement.

« Il aurait pu être tour à tour commissaire-priseur, spécialiste de l'art africain, expert en céramique japonaise, chimiste, biochimiste, informaticien, courtier en Lalique, moniteur de pêche à la mouche, chef dans le célèbre "Chez Jojo", spécialiste des mélanges improbables, il a été tout ça à la fois

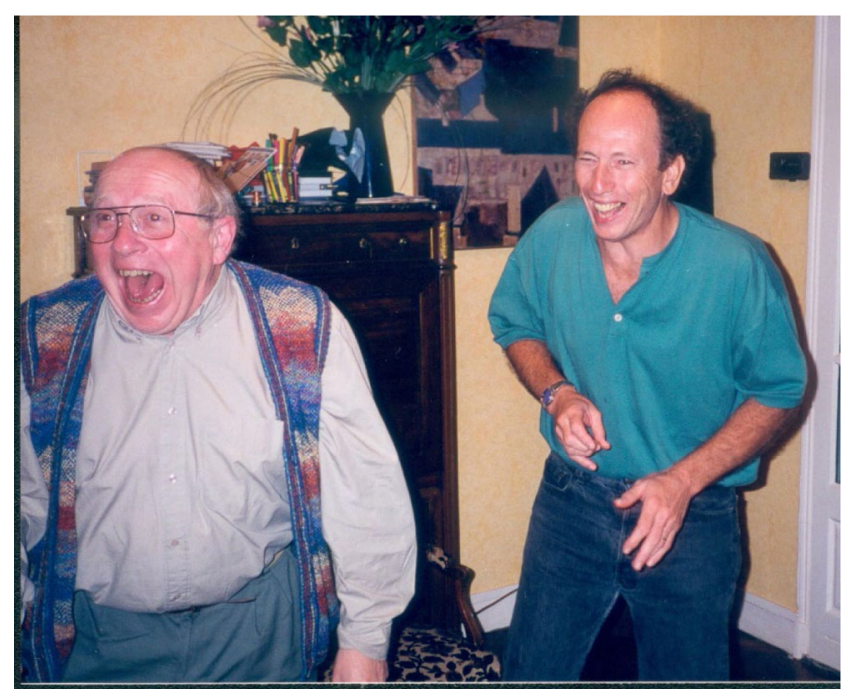

Fig. 1 Après les « manips »... 
tout en étant un chercheur extraordinaire, curieux de tout, voulant à chaque fois comprendre, s'intéressant plus aux mécanismes qu'aux résultats ; un passeur de connaissances, patient avec ses élèves, ne comptant pas son temps. »

«Je fais partie des quelques privilégiés qui ont eu la chance de partager avec toi une année de DEA dans ce bureau de la faculté de médecine Xavier-Bichat. Depuis, « l'étudiant», comme tu aimais encore à m'appeler, a un peu mûri, mais je garde en souvenirs ces soirées où tu nous expliquais ta chasse online pour dénicher des objets improbables : masques africains, objets de cuisine ou autres céramiques dont tu connaissais tous les secrets de fabrication. »

« L'étonnement, le plaisir et le rire, ce sont probablement les mots qui définissent le mieux mon état d'esprit pour ces nombreux moments passés à tes côtés depuis ces 15 dernières années. Ton regard aiguisé, ta curiosité et ta capacité à faire systématiquement émerger les éléments pertinents et très souvent drôles de situations pouvant paraittre banales sont pour moi une source inépuisable de plaisir. "Chaque jour, une découverte" est je crois la phrase la plus représentative de qui tu es. Sans le moindre épuisement, tout au long de ta vie, tu as gardé ce plaisir de découvrir, d'explorer, de te passionner pour des choses parfois improbables et surtout ce plaisir d'accompagner des étudiants. J'ai eu la chance d'en faire partie, et tu es l'une des rencontres qui m'ont construit. »

" Les années passées à tes côtés comme étudiant en DEA puis en thèse ont été un parcours initiatique d'une exceptionnelle richesse scientifique et humaine. Ton humour décapant et décalé, ton indépendance d'esprit, ton intelligence critique m'ont profondément marqué, tout autant que ta bienveillance à mon égard. Des découvertes (cf. la devise rapportée ci-avant), il y en eut pour moi dans de nombreux domai- nes : physiologie, médecine, botanique, bricolage, physique, chimie, programmation informatique, biostatistiques... sans parler des dessins de Cabu ou des répliques cultes des Shadocks ("plus ça rate, plus il y a de chances que ça marche"). Toutes ces disciplines s'articulaient entre elles avec une fluidité naturelle et, toujours, une liberté d'esprit et une honnêteté intellectuelle que tu as je crois transmises à tous ceux qui t'ont côtoyé. Merci Georges pour ce que tu nous as transmis. »

Nous avons tous eu la chance de devenir les amis de Georges ainsi que de Michèle, son épouse, si souriante et chaleureuse.

Nos pensées vont vers elle, leurs deux enfants Christine et Frédéric et vers leurs petits-enfants.

Nous avons mis sur son cercueil une de ses BD de Reiser préférées : "La vie au grand air ». Tout un programme.

Adieu Georges et merci. Nous t'embrassons.

Didier Dreyfuss, au nom de tous les élèves de Georges Saumon.

\section{Références}

1. Dreyfuss D, (2009) Mon papier 20 ans après ou comment j'ai fait de la recherche translationnelle sans le savoir. Réanimation 18: 160-162

2. Webb HH, Tierney DF, (1974) Experimental pulmonary edema due to intermittent positive pressure ventilation with high inflation pressures. Protection by positive end-expiratory pressure. Am Rev Respir Dis 110: 556-565

3. Tierney DF, (2003) Ventilator-induced lung injury occurs in rats, but does it occur in humans? Am J Respir Crit Care Med 168: $1414-1415$ 